\title{
Moiré-Modulated Conductance of Hexagonal Boron Nitride Tunnel Barriers
}

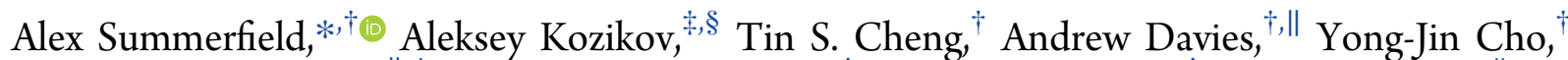
Andrei N. Khlobystov, ${ }^{\|, \perp}$ Christopher J. Mellor, ${ }^{\dagger}$ C. Thomas Foxon, ${ }^{\dagger}$ Kenji Watanabe, \#®

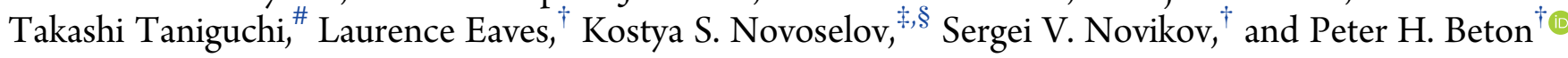

${ }^{\dagger}$ School of Physics and Astronomy, University of Nottingham, Nottingham, NG7 2RD, United Kingdom

${ }^{\ddagger}$ School of Physics and Astronomy and ${ }^{\S}$ National Graphene Institute, University of Manchester, Oxford Road, Manchester, M13 9PL, United Kingdom

${ }^{\|}$School of Chemistry and ${ }^{\perp}$ Nottingham Nanoscale and Microscale Research Centre, University of Nottingham, Nottingham, NG7 2RD, United Kingdom

${ }^{\#}$ National Institute for Materials Science, 1-1 Namiki, Tsukuba, Ibraki 305-0044, Japan

Supporting Information

ABSTRACT: Monolayer hexagonal boron nitride (hBN) tunnel barriers investigated using conductive atomic force microscopy reveal moire patterns in the spatial maps of their tunnel conductance consistent with the formation of a moire superlattice between the $\mathrm{hBN}$ and an underlying highly ordered pyrolytic graphite (HOPG) substrate. This variation is attributed to a periodc modulation of the local density of states and occurs for both exfoliated hBN barriers and epitaxially grown layers. The epitaxial barriers also exhibit enhanced conductance at localized subnanometer regions which are attributed to exposure of the substrate to a nitrogen plasma source during the high temperature growth process. Our results show clearly a spatial periodicity of tunnel current due to the formation of a moiré superlattice and we argue that this can provide a mechanism for elastic scattering of charge carriers for similar interfaces embedded in graphene $/ \mathrm{hBN}$ resonant tunnel diodes.

KEYWORDS: Boron nitride, epitaxy, growth, tunneling, superlattice, moire, heterostructure
T exagonal boron nitride (hBN) has emerged as one of the 1 most widely investigated ${ }^{1}$ two-dimensional (2D) materials (2DMs) since the initial isolation of graphene. ${ }^{2}$ It is an insulator with a large energy gap of $\sim 6 \mathrm{eV}$ and is isostructural with graphene with a lattice mismatch of only $\sim 1.8 \%$ between the two crystals. ${ }^{1}$ Heterostructures based on the two crystals represent a new class of functional electronic materials. ${ }^{3-9}$ For example, graphene mounted on the atomically flat surface of hBN has a much higher carrier mobility due to the reduction of potential fluctuations encountered in early graphene devices fabricated on $\mathrm{SiO}_{2}$. ${ }^{1,10-12}$ When the crystal lattice of graphene is overlaid on $\mathrm{hBN}$, an additional physical property emerges, namely the formation of a hexagonal moire fringe pattern with an associated superlattice potential which leads to pronounced changes in the in-plane magnetoconductivity of graphene through the formation of mini-bands and small energy gaps. ${ }^{3,5,8,13-18}$ Moiré patterns might also be expected when $\mathrm{hBN}$ layers are placed or grown on graphene/graphite. However, there have been very few reports of the formation of such structures ${ }^{19-21}$ although these interfaces form an integral part of graphene/hBN resonant tunneling diodes and related van der Waals heterostructures. ${ }^{4,19,22-24}$ In particular, $\mathrm{hBN}$ tunnel barriers have been exploited to produce resonant tunneling transistors with gate-controlled negative differential conductance. $^{22,25-29}$

In this paper, we investigate the influence of the formation of a moire pattern on the tunneling conductance of a hBN barrier. Using conductive atomic force microscopy (cAFM), we show that there is a pronounced spatial variation in the tunnel current which we attribute to the moire pattern formed at an $\mathrm{hBN} /$ graphite interface. The effect is observed both in heterostructures formed by placement of exfoliated $\mathrm{hBN}$, similar to the process used in the fabrication of most resonant tunneling diodes, ${ }^{30-33}$ and also in the interface formed between epitaxially grown $\mathrm{hBN}$ layers and a graphite substrate.

The hBN/graphite tunnel barrier devices investigated here were fabricated using the "dry-transfer" method ${ }^{34}$ and also from hBN grown epitaxially on highly oriented pyrolytic graphite (HOPG) using plasma-assisted molecular beam epitaxy (PA-MBE) following protocols which we have recently reported. ${ }^{19-21}$ Samples were then imaged using a combination of AFM/cAFM and scanning tunneling microscopy (STM) in

Received: March 26, 2018

Revised: June 13, 2018

Published: June 18, 2018 
ambient conditions. The details of the fabrication and imaging procedures for these devices are presented in the Supporting Information (SI).

Figure 1a shows a schematic diagram of the exfoliated hBN device. The hBN flake is placed on an exfoliated (multilayer)

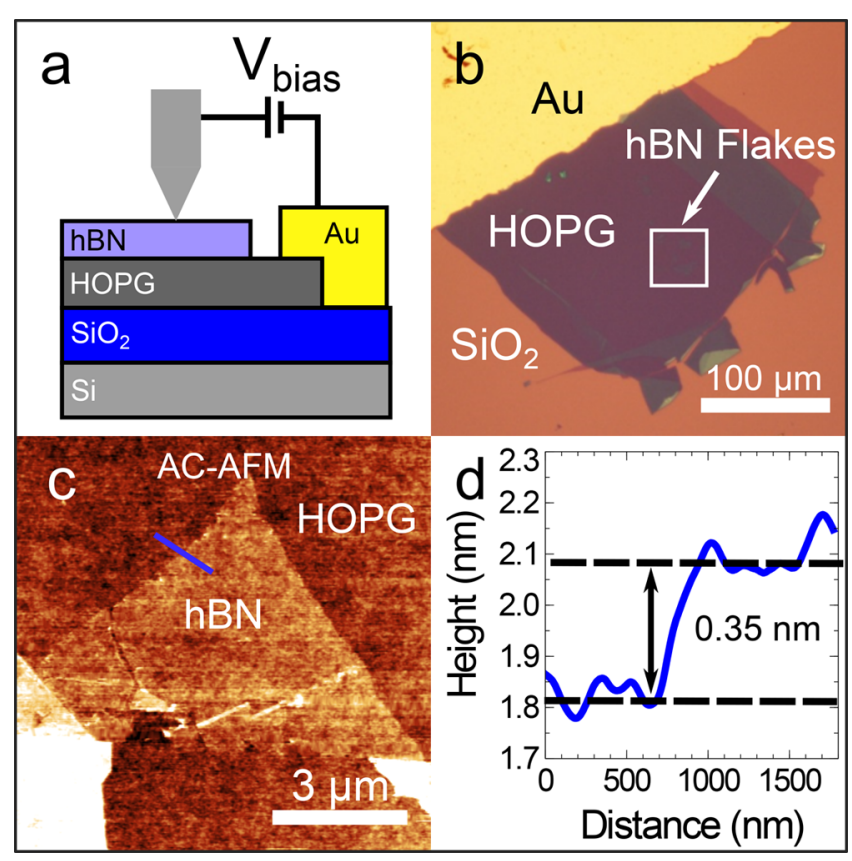

Figure 1. (a) Schematic of exfoliated hBN/HOPG tunnel barrier device. (b) Bright-field optical image of the device. The white box indicates the approximate position of the exfoliated hBN flakes. Darkfield optical imaging of this region is given in the Supporting Information, see Figure S1. (c) AC-mode AFM image of part of the region indicated by the white box in image (b), showing a monolayer hBN flake. (d) Line profile along the region indicated by the blue line in (c) demonstrating the monolayer thickness of the exfoliated hBN flake.

HOPG layer mounted on a $\mathrm{Si} / \mathrm{SiO}_{2}$ wafer (oxide thickness $300 \mathrm{~nm}$ ). A thermally evaporated $\mathrm{Cr} / \mathrm{Au}$ contact is deposited on the HOPG and acts as a counter-electrode to the cAFM tip. An optical micrograph of the HOPG flake and $\mathrm{Cr} / \mathrm{Au}$ electrode structure is shown in Figure $1 \mathrm{~b}$. The top surface of the flake is covered in exfoliated hBN flakes which cannot be resolved in bright-field optical microscopy, so dark-field optical microscopy is used to highlight the edges of the flakes (see SI Figure S1b). A monolayer-thick (ML) hBN flake (see Figure 1c) was selected by performing large area tapping-mode (AC-mode) AFM scans to identify thin flakes. The thickness of the hBN flake, as shown in Figure 1d, was $0.35 \pm 0.05 \mathrm{~nm}$, close to the expected value for $1 \mathrm{ML}$ of $\mathrm{hBN}$.

Figure 2 presents cAFM data acquired on the exfoliated device shown in Figure 1; the applied voltage between tip and substrate is $10 \mathrm{mV}$. Figure $2 \mathrm{a}, \mathrm{b}$ shows simultaneously acquired contact-mode topography (Figure 2a) and current channel (Figure 2b) images of the same region on the ML hBN flake. The surface has a low roughness as indicated by the red line profile in Figure $2 \mathrm{c}$ with no distinct features such as step edges/gaps on the hBN surface; any residual surface roughness is likely due to the underlying $\mathrm{SiO}_{2}$ support substrate. However, the tunnel current image (Figure 2b) clearly shows an $11.7 \pm$ $0.1 \mathrm{~nm}$ period moire pattern which is detectable over the entire surface of the $\mathrm{hBN}$ flake, indicative of a single crystalline

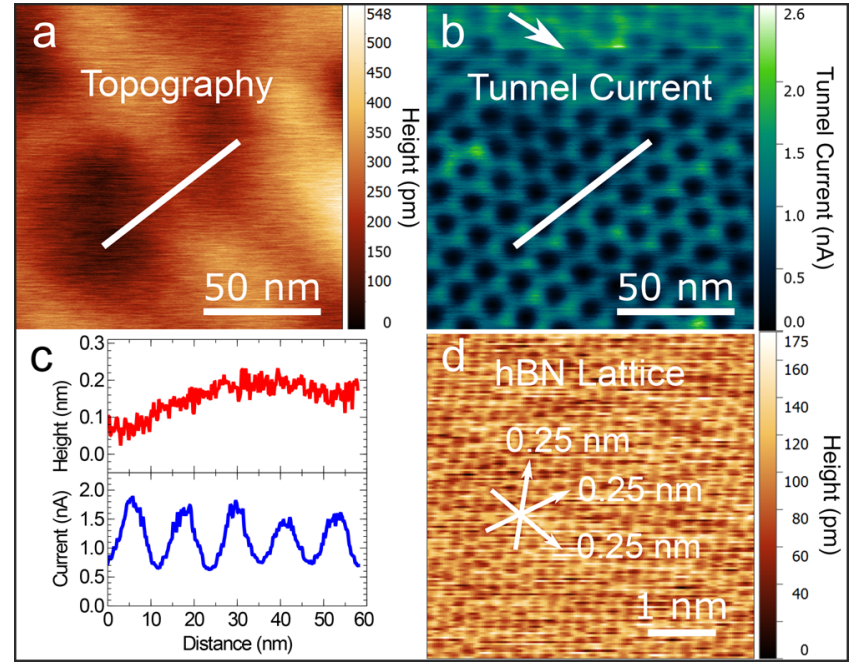

Figure 2. cAFM images of the exfoliated hBN/HOPG device shown in Figure 1. (a) Height-channel image of the surface topography of hBN. (b) Current map of the same area as in image (a), exhibiting an $11.7 \mathrm{~nm}$ moire pattern due to rotational misalignment between the hBN and HOPG lattices (cAFM tip bias: $+10 \mathrm{mV}$ ). (c) (top) Height profile along the line indicated in (a). (bottom) Conductance profile along the line indicated in (b) showing a spatial variation in tunnel current across the hBN surface (bottom). (d) Contact mode lattice image showing the hBN lattice and expected hBN periodicity.

domain. Using a simple geometric relationship ${ }^{3}$ and assuming that the $\mathrm{hBN}$ is unstrained, we calculate a rotational mismatch of $0.66 \pm 0.02^{\circ}$ between the HOPG and $\mathrm{hBN}$ lattices from the measured moiré periodicity with an expected moiré twist angle of $27.5^{\circ}$ relative to the graphite lattice. We have separately confirmed that the hBN and HOPG lattices are aligned by performing high-speed contact-mode imaging on the hBN flake and HOPG surface near the hBN flake (see Figure S4b,c). Measurement of the twist angle of the moire pattern relative to the HOPG lattice $\left(27 \pm 1^{\circ}\right)$ also agrees with the expected angle calculated above (Figure S4d). The formation of the moire pattern results in a periodic decrease of $\sim 50 \%$ from the peak value of tunnel current along the principal axis of the hexagonal moire pattern, as shown by the blue line profile in Figure $2 \mathrm{c}$ with no correlation with the height profile in Figure 2a. Figure $2 \mathrm{~d}$ also shows atomic lattice imaging of the $\mathrm{hBN}$ surface (acquired in contact mode using high speed scanning, see SI) which confirms the expected lattice spacing of $0.25 \pm 0.01 \mathrm{~nm}$ for $\mathrm{hBN} .^{35}$

A schematic of the PA-MBE hBN tunnel barriers is shown in Figure 3a. As with the exfoliated device, the bulk HOPG substrate provides a counter electrode to the cAFM tip and is electrically connected using conducting silver paint. Following growth (see SI), the HOPG surface is almost completely covered in monolayer-thick hBN (and some regions of bilayer (BL) and multilayer hBN around HOPG step-edges). Optically, the sample has a uniform contrast when observed using bright field microscopy and no surface detail due to the growth is observed (see Figure S1a). The contact-mode AFM image of the surface topography of the PA-MBE hBN in Figure $3 \mathrm{~b}$ shows the laterally polycrystalline $\mathrm{ML} \mathrm{hBN}$ domains for a sample with a high surface coverage. The expected $\sim 0.35 \mathrm{~nm}$ step height of ML hBN is observed in measurements at the edge between ML hBN and uncovered HOPG (see Figure 3c), in agreement with our previous AFM measurements of MBE-grown $\mathrm{hBN}$ on HOPG. ${ }^{19,20}$ 


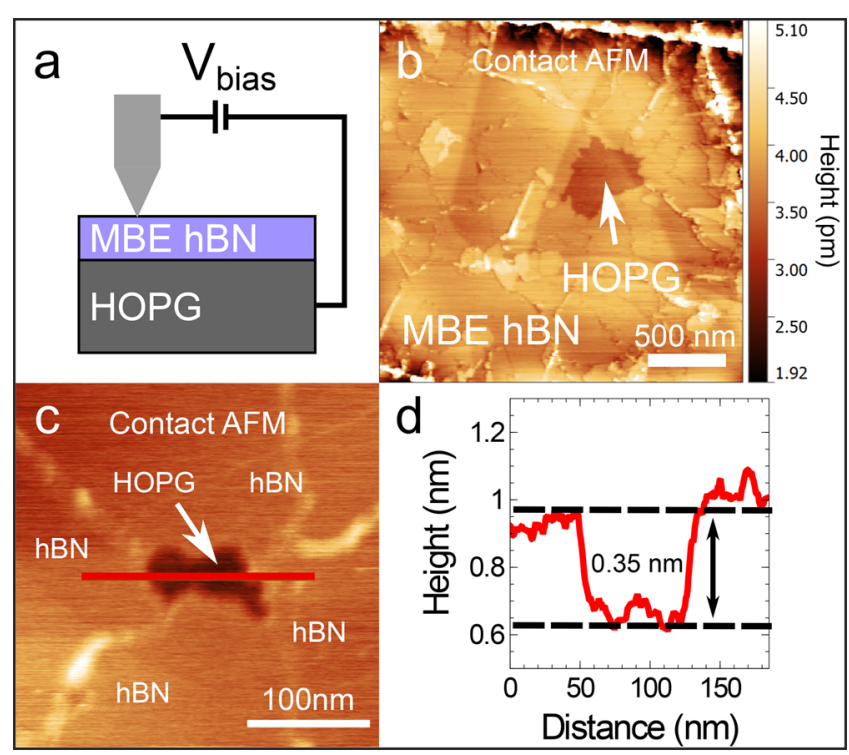

Figure 3. (a) Schematic of PA-MBE hBN/HOPG device and the experimental setup for cAFM imaging. (b) Contact mode AFM image of MBE hBN on HOPG; the arrow indicates a region of HOPG not covered by PA-hBN growth. (c) Contact mode AFM images of multiple hBN domains around a small region of HOPG not covered by $\mathrm{hBN}$ growth (dark indicated area). (d) Height profile along the red line in (c) indicating the monolayer hBN thickness.

Figure 4 presents CAFM data for the device with PA-MBEgrown hBN on HOPG. Figure 4a shows the topography of a region consisting of several $\mathrm{hBN}$ domains. The central region with darker contrast (i.e., topographically lower) is the HOPG substrate (labeled) which has remained uncovered by hBN growth. Figure $4 \mathrm{~b}$ shows a cAFM image for the top half of the region in Figure 4a. The line profiles along this central region (blue and red lines in Figure $4 a, b$ ) are plotted in the inset of Figure $4 \mathrm{a}$ where exposed HOPG is present indicates that the uncovered HOPG has significantly higher tip-sample junction conductance, as expected. Also indicated in both Figure 4a,b are the positions where images in panels $\mathrm{c}$ and e in Figure 4 were acquired. High-resolution images of the hBN domains are shown in Figure 4c,e. In some domains, we observe moiré patterns in the tunnel conductance signal. An example is shown in Figure $4 c$ in which a spatial variation of tunnel current with a period of $13.5 \pm 0.1 \mathrm{~nm}$ is observed, indicating a small rotational mismatch of $0.25 \pm 0.04^{\circ}$ between the $\mathrm{hBN}$ and HOPG lattices. ${ }^{3}$ A line-profile across the moire pattern along this feature (Figure $4 \mathrm{~d}$ ) shows an $\sim 50 \%$ decrease in the tunnel current from the maximum value along the moire fringes, similar to the variation observed in the measurements of the exfoliated hBN device. In addition to the periodic variation, some localized regions of higher surface conductance are observed which are discussed later.

Figure $4 \mathrm{e}$ shows one $\mathrm{hBN}$ domain in which no moire pattern is resolved. This suggests that any moiré pattern present has either a period or amplitude which is too small to resolve, possibly due to the misalignment of $\mathrm{hBN}$ and underlying HOPG. The current spikes in the conductance profile and bright features in the cAFM current maps (Figure $4 c-e$ ) are consistent across several scans and are observed both on hBN domains that show moiré fringes and also those on which no moire pattern is resolved, indicating localized regions of high surface conductance. Analysis of the increase in conductance at these spots (see Figure S2) shows a typical 2- to 3-fold increase relative to the average conductance of the whole cAFM image for the regions shown in Figure 4c,e respectively, with some regions showing increases as high as a factor of $\sim 13$.

High-resolution lattice imaging (and simultaneous acquisition of tunnel current signal; Figure $4 \mathrm{~g}, \mathrm{~h}$ ) of the $\mathrm{hBN}$ lattice around these regions of high conductance yield the periodicity of the hBN lattice $(0.25 \pm 0.01 \mathrm{~nm})$ as is the case for the exfoliated device shown in Figure 2. This allows us to image the individual regions of high conductance and shows that they are localized around an area of approximately one lattice site, although we do not observe any lattice vacancies or defects in contact mode images of the hBN layer in this region (Figure $4 \mathrm{~g}$ ). As no vacancy is observed in the lattice of the PA-MBE hBN layer using topographic imaging, we speculate that the highly conducting regions observed in our MBE hBN samples may be caused by defects in the underlying HOPG substrate, introduced, for example, by exposure of the HOPG substrate to a $\mathrm{N}_{2}$ plasma during the growth process. ${ }^{36}$ In order to test this hypothesis, we imaged the regions of HOPG not covered by hBN growth with cAFM. A current map of a region of exposed HOPG is shown in Figure $4 \mathrm{f}$ and reveals a non-uniform current density profile, similar to the region in Figure $4 \mathrm{e}$ with $\mathrm{ML} \mathrm{hBN}$ coverage. For these regions, however, we were unable to achieve lattice-level resolution in both height and current-channels. To further investigate the effect of $\mathrm{N}_{2}$ plasma on the HOPG surface, we subjected clean HOPG substrates to the same hightemperature conditions used to grow the PA-MBE sample shown in Figures 3 and 4, both with and without the presence of an $\mathrm{N}_{2}$ plasma. cAFM images of these samples (Figure S3a,d) show clearly that defects are formed on the surface through exposure to the $\mathrm{N}_{2}$ plasma rather than high temperature annealing alone.

Because the formation of a moire superlattice between $\mathrm{hBN}$ and graphite modifies the local density of states (LDOS) of the hBN layer, we performed ambient STM imaging in order to confirm our cAFM observations. STM can typically provide better contrast than CAFM as it is highly sensitive to variations in surface LDOS and has potentially higher lateral resolution than AFM in ambient conditions. Figure 5 presents constantcurrent STM images of the PA-MBE hBN surface. As expected, large-area STM images of the PA-MBE hBN surface show that multiple domains of $\mathrm{ML} \mathrm{hBN}$ and some regions of $\mathrm{BL} \mathrm{hBN}$ are present; these are shown in Figure 5a. Several different surface features appear in the main image which confirm the laterally polycrystalline nature of the grown $\mathrm{hBN}$ layers; these are revealed in more detail in the further subfigures. In addition, the STM images show that a range of moire periodicities are present, confirming that there are multiple rotational alignments with the substrate. ${ }^{3,10}$ Figure $5 \mathrm{~b}$ shows two neighboring hBN domains which exhibit moire patterns of differing rotational alignment with the underlying HOPG, the left domain having a moire period of $\sim 16 \mathrm{~nm}$ and the right domain of $\sim 14 \mathrm{~nm}$, indicating that both of these domains are closely aligned with the HOPG lattice. Interestingly, the larger moire period $(\sim 16 \mathrm{~nm})$ region has a periodicity greater than the maximum moire period expected for aligned HBN and HOPG lattices $(\sim 14 \mathrm{~nm})$. This indicates that the $\mathrm{hBN}$ lattice is compressively strained by $\sim 0.24 \%$ in this region, assuming that the $\mathrm{hBN}$ and graphite lattices are perfectly aligned. ${ }^{13,14,37-39}$

Figure $5 \mathrm{c}$ and associated line profiles in Figure $5 \mathrm{j}$ confirm the presence of the small, high-conductance regions on the $\mathrm{hBN}$ surface in our STM images. This supports our cAFM observations in Figure 4. Figure 5d-i shows images of both $\mathrm{ML}$ and $\mathrm{BL}$ 


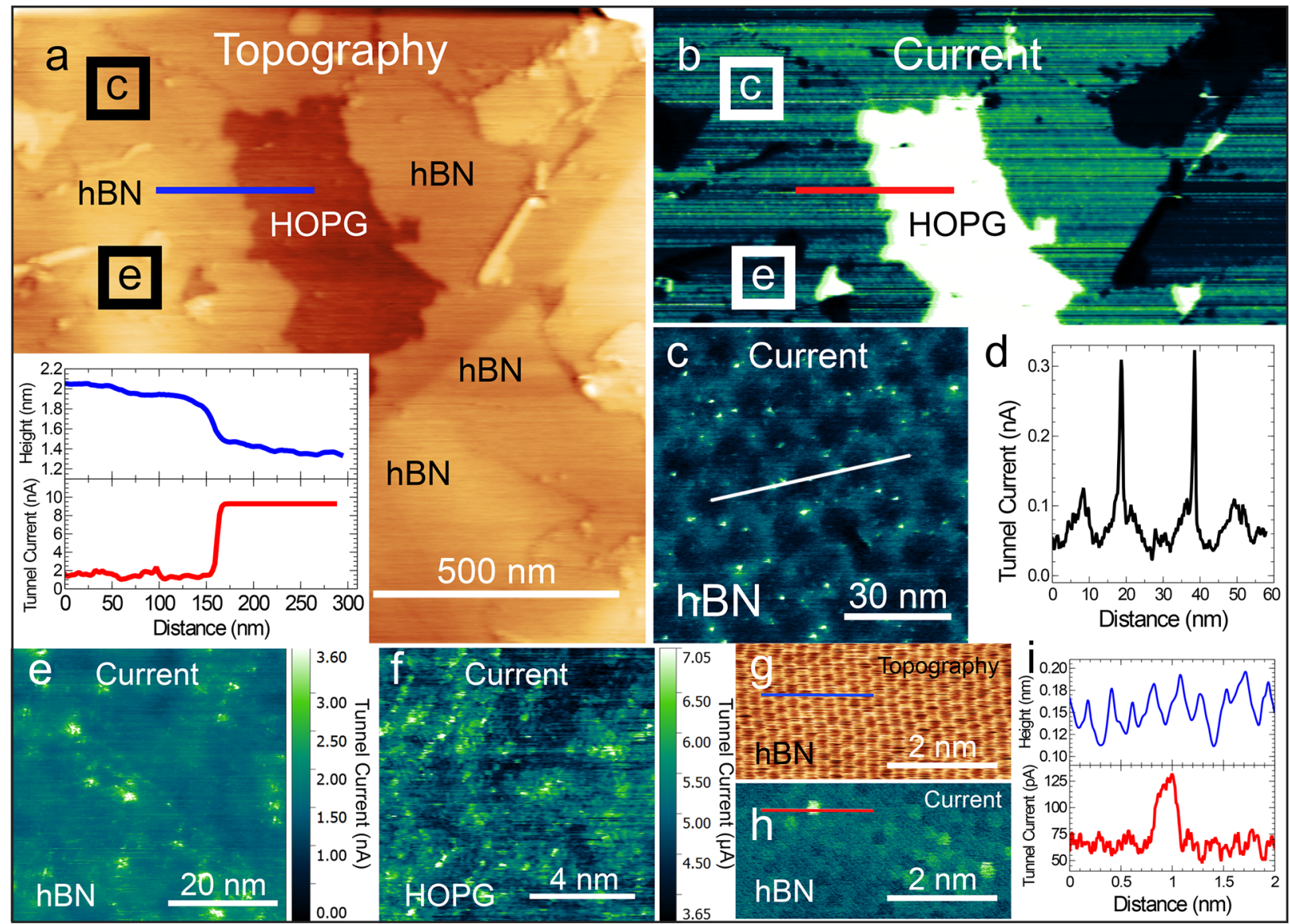

Figure 4. Topographic and cAFM images of PA-MBE grown hBN on HOPG; the label in each image indicates the imaging mode (top label) and/or the surface being imaged (bottom left label). (a) Contact-mode AFM image showing PA-MBE hBN domains with an exposed area of HOPG indicted by the labels. The boxes show the positions of the high-resolution scans of the hBN domains for images (c,e). (inset) Line profiles along the regions indicated by the blue and red lines in $(a, b)$ respectively at an interface between PA-MBE hBN and exposed HOPG. (b) Current channel image of the top half of (a). The labeled boxes correspond to the same regions labeled in (a), showing where high resolutions scans were taken. (c) Tunnel current map of the region indicated by the topmost white box in image (b) showing a moire pattern with a period of $13.5 \mathrm{~nm}$. (d) Line profile across the white line in image (c) showing the variation in tunnel current. (e) Tunnel current channel image of non-aligned PA-hBN exhibiting no moire pattern in the current signal. (f) Current map of an exposed HOPG region with no hBN overgrowth. (g) Contact mode AFM image showing the hBN lattice. (h) Tunnel current channel of (b) showing localized hot-spots of increased conductance. (i) Height profile (top) and tunnel current profile (bottom) along the blue and red lines marked in (g,h) respectively. All cAFM images were taken with a tip bias of $+100 \mathrm{mV}$ with the exception of $(\mathrm{h})$ which was acquired using a tip bias of $+10 \mathrm{mV}$.

regions of hBN that have a common rotational alignment. Figure $5 \mathrm{~d}$ shows a BL hBN island (darker contrast due to reduced tunnel conduction through the thicker $\mathrm{hBN}$ ) surrounded by ML hBN. High-resolution imaging of both the ML and BL islands (Figure 5e,g) show that both regions exhibit aligned, short-period moire patterns with a periodicity of $2.4 \pm 0.01 \mathrm{~nm}$, suggesting that the $\mathrm{BL}$ region is growing epitaxially on the ML hBN with a common misalignment of $5.8 \pm 0.3^{\circ}$ to the underlying HOPG. ${ }^{3}$ Further imaging of another epitaxial $\mathrm{BL}$ region neighboring the ML region in Figure $5 \mathrm{~d}$ is shown in Figure 5i, this area also exhibits a moiré pattern aligned with the ML region with the same $2.4 \mathrm{~nm}$ moire periodicity, indicating that they also share a common rotational alignment with the HOPG substrate. It is possible that these regions with smaller moiré periods correspond to areas where no moiré pattern is resolved in cAFM images.

Our measurements reveal the presence of local electronic moiré effects in hBN tunnel barriers due to rotational misalignment of both exfoliated and PA-MBE-grown hBN on HOPG. The periodic current modulation which we observe in cAFM implies that tunneling electrons in a $\mathrm{hBN} /$ graphene heterostructure experience a variation in potential landscape with a period equal to that of the moire pattern. It might be anticipated that this periodic variation would provide a source of elastic scattering ${ }^{40}$ in which the in-plane wave vector, $\mathbf{k}$, of a tunneling electron would be scattered by $\mathbf{k}_{M}^{i}(i=1,2)$, the reciprocal lattice vector of the moire pattern, and this mechanism may also be relevant to the buried interfaces formed in graphene/ hBN resonant tunneling diodes. ${ }^{22}$

The topographic structure of the two types of hBN tunnel barrier studied here differ significantly from each other. Nevertheless, despite some differences in their microscopic structure, cAFM imaging of ML hBN shows broadly the same tunnel current behavior, with both samples exhibiting hexagonal moiré patterns. In addition, for both exfoliated and PA-MBE grown devices, our maps of tunnel current reveal a similar variation in the tunnel current along the principal axes of the moire pattern. The observation of a moire pattern with a periodicity larger than expected for aligned hBN on graphite is particularly interesting because it implies that in these areas the lattice mismatch between the $\mathrm{hBN}$ and graphite must be reduced which would be consistent with a compressive strain in the epitaxial growth layer. 


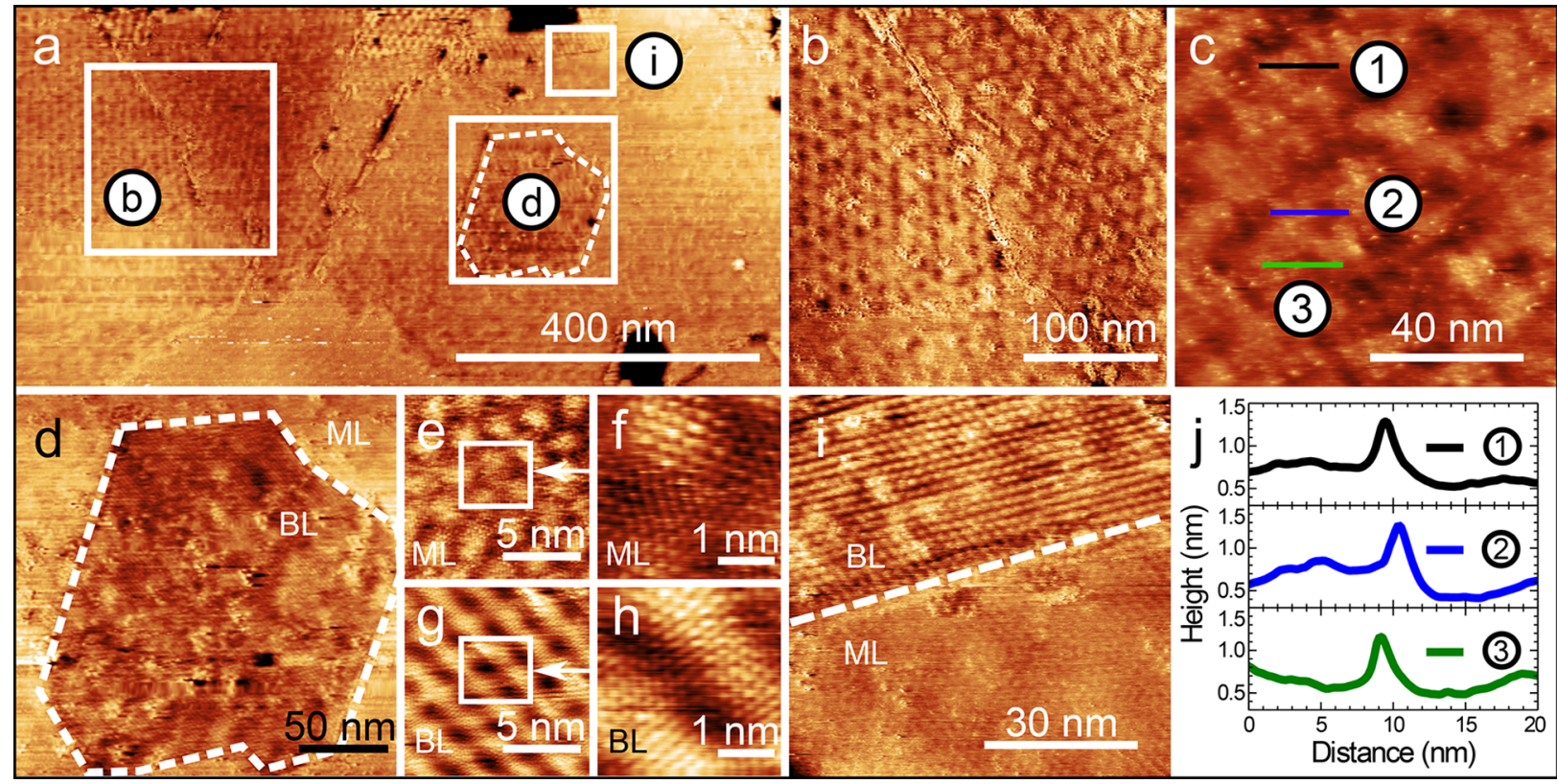

Figure 5. Constant current STM images of hBN grown on HOPG using PA-MBE. (a) STM image showing multiple domains of hBN grown on HOPG. The white boxes indicate the regions shown in images (b,d,i), respectively. (b) Moire patterns visible on either side of the boundary between two hBN domains, indicated by the white box in (a). The moire periods on the left and right domains are 16 and $14 \mathrm{~nm}$, respectively. (c) STM image of a monolayer hBN domain on HOPG without moiré patterns showing small regions of increased brightness (i.e., higher STM topography). The profiles indicated by the colored lines are shown in (j). (d) STM image of a bilayer (BL) hBN domain surrounded by monolayer (ML) hBN as indicated by the box in (a). (e) Moire patterns visible on ML hBN region in (d). (f) Zoom of (e) showing lattice-level contrast of ML hBN domain. (g) STM image of moiré patterns visible on BL hBN domain shown in (d). (h) Zoom of (g) showing lattice-level contrast of BL hBN. (i) STM image showing border between ML and BL hBN as indicated by the white box in (a). In both regions, moire patterns are visible and have a periodicity of $2.4 \pm 0.1 \mathrm{~nm}$ for both the $\mathrm{ML}$ and BL regions, respectively. (j) Constant current profiles along the lines indicated in (c) showing small regions with increased tunnel conductance.

Our observations of an increased localized conductance in the MBE-grown hBN samples, which we attribute to plasmainduced defects in the HOPG, show that the effects of substrate damage need to be considered in the future fabrication of 2DM vdW heterostructures grown using plasma-assisted methods. We plan to follow up these observations with studies using a combination of low-temperature UHV AFM and STM measurements in order to fully characterize the electronic behavior of these defects. Overall, our results show that moiré effects, which have been demonstrated to lead to pronounced changes in the properties of graphene on $\mathrm{hBN}$, also lead to significant modifications of the electronic properties of the inverse structures, namely hBN on graphene. These differences are present in both the epitaxial and exfoliated devices, thus offering the prospect of high-quality epitaxial materials by growing at high temperatures using PA-MBE. This technique could provide a platform for the growth of hBN/ graphene heterostructures in which strain and moire pattern modulation of electronic properties can be introduced in a controlled way.

\section{ASSOCIATED CONTENT}

\section{S Supporting Information}

The Supporting Information is available free of charge on the ACS Publications website at DOI: 10.1021/acs.nanolett.8b01223.

Experimental and growth methodologies for both $\mathrm{MBE}$ and exfoliated hBN tunnel barriers; optical images of hBN samples; histogram of conductance spikes in $\mathrm{MBE}$ hBN devices; cAFM imaging of annealed and $\mathrm{N}_{2}$ plasma treated HOPG samples (PDF)

\section{AUTHOR INFORMATION}

\section{Corresponding Author}

*E-mail: alex.summerfield@nottingham.ac.uk.

ORCID

Alex Summerfield: 0000-0001-6091-3969

Andrei N. Khlobystov: 0000-0001-7738-4098

Kenji Watanabe: 0000-0003-3701-8119

Peter H. Beton: 0000-0002-2120-8033

\section{Notes}

The authors declare no competing financial interest.

All data on which this paper is based may be publicly accessed at http://rdmc.nottingham.ac.uk/ under doi.org/10.17639/ nott.358.

\section{ACKNOWLEDGMENTS}

This work was supported by the Engineering and Physical Sciences Research Council [grant number(s) EP/L013908/1, EP/P019080/1, DTP Grant EP/M50810X/1]; the Leverhulme Trust [grant number RPG-2014-129]; the EU Graphene Flagship Program; the European Research Council Synergy Grant Hetero2D; The Royal Society and the U.S. Army Research Office (W911NF-16-1-0279), K.W. and T.T. acknowledge support from the Elemental Strategy Initiative conducted by the MEXT, Japan and the CREST (JPMJCR15F3).

\section{REFERENCES}

(1) Dean, C. R.; Young, A. F.; Meric, I.; Lee, C.; Wang, L.; Sorgenfrei, S.; Watanabe, K.; Taniguchi, T.; Kim, P.; Shepard, K. L.; Hone, J. Nat. Nanotechnol. 2010, 5, 722-726. 
(2) Novoselov, K. S.; Geim, A. K.; Morozov, S. V.; Jiang, D.; Zhang, Y.; Dubonos, S. V.; Grigorieva, I. V.; Firsov, A. A. Science 2004, 306, 666-669.

(3) Yankowitz, M.; Xue, J.; Cormode, D.; Sanchez-Yamagishi, J. D.; Watanabe, K.; Taniguchi, T.; Jarillo-Herrero, P.; Jacquod, P.; LeRoy, B. J. Nat. Phys. 2012, 8, 382-386.

(4) Britnell, L.; Gorbachev, R. V.; Jalil, R.; Belle, B. D.; Schedin, F.; Mishchenko, A.; Georgiou, T.; Katsnelson, M. I.; Eaves, L.; Morozov, S. V.; Peres, N. M. R.; Leist, J.; Geim, A. K.; Novoselov, K. S.; Ponomarenko, L. A. Science 2012, 335, 947-950.

(5) Dean, C. R.; Wang, L.; Maher, P.; Forsythe, C.; Ghahari, F.; Gao, Y.; Katoch, J.; Ishigami, M.; Moon, P.; Koshino, M.; Taniguchi, T.; Watanabe, K.; Shepard, K. L.; Hone, J.; Kim, P. Nature 2013, 497, $598-602$.

(6) Eckmann, A.; Park, J.; Yang, H.; Elias, D.; Mayorov, A. S.; Yu, G.; Jalil, R.; Novoselov, K. S.; Gorbachev, R. V.; Lazzeri, M.; Geim, A. K.; Casiraghi, C. Nano Lett. 2013, 13, 5242-5246.

(7) Wang, L.; Meric, I.; Huang, P. Y.; Gao, Q.; Gao, Y.; Tran, H.; Taniguchi, T.; Watanabe, K.; Campos, L. M.; Muller, D. A.; Guo, J.; Kim, P.; Hone, J.; Shepard, K. L.; Dean, C. R. Science 2013, 342, 614617.

(8) Ponomarenko, L. A.; Gorbachev, R. V.; Yu, G. L.; Elias, D. C.; Jalil, R.; Patel, A. A.; Mishchenko, A.; Mayorov, A. S.; Woods, C. R.; Wallbank, J. R.; Mucha-Kruczynski, M.; Piot, B. A.; Potemski, M.; Grigorieva, I. V.; Novoselov, K. S.; Guinea, F.; Fal'ko, V. I.; Geim, A. K. Nature 2013, 497, 594-597.

(9) Mayorov, A. S.; Gorbachev, R. V.; Morozov, S. V.; Britnell, L.; Jalil, R.; Ponomarenko, L. A.; Blake, P.; Novoselov, K. S.; Watanabe, K.; Taniguchi, T.; Geim, A. K. Nano Lett. 2011, 11, 2396-2399.

(10) Decker, R.; Wang, Y.; Brar, V. W.; Regan, W.; Tsai, H. Z.; Wu, Q.; Gannett, W.; Zettl, A.; Crommie, M. F. Nano Lett. 2011, 11, 2291-2295.

(11) Xue, J.; Sanchez-Yamagishi, J.; Bulmash, D.; Jacquod, P.; Deshpande, A.; Watanabe, K.; Taniguchi, T.; Jarillo-Herrero, P.; Leroy, B. J. Nat. Mater. 2011, 10, 282-285.

(12) Das Sarma, S.; Hwang, E. H. Phys. Rev. B: Condens. Matter Mater. Phys. 2011, 83, 121405.

(13) Woods, C. R.; Britnell, L.; Eckmann, A.; Ma, R. S.; Lu, J. C.; Guo, H. M.; Lin, X.; Yu, G. L.; Cao, Y.; Gorbachev, R. V.; Kretinin, A. V.; Park, J.; Ponomarenko, L. A.; Katsnelson, M. I.; Gornostyrev, Y. N.; Watanabe, K.; Taniguchi, T.; Casiraghi, C.; Gao, H.; Geim, A. K.; Novoselov, K. S. Nat. Phys. 2014, 10, 451-456.

(14) Summerfield, A.; Davies, A.; Cheng, T. S.; Korolkov, V. V.; Cho, Y.; Mellor, C. J.; Foxon, C. T.; Khlobystov, A. N.; Watanabe, K.; Taniguchi, T.; Eaves, L.; Novikov, S. V.; Beton, P. H. Sci. Rep. 2016, 6, 22440.

(15) Tang, S.; Wang, H.; Zhang, Y.; Li, A.; Xie, H.; Liu, X.; Liu, L.; Li, T.; Huang, F.; Xie, X.; Jiang, M. Sci. Rep. 2013, 3, 2666.

(16) Hunt, B.; Sanchez-Yamagishi, J. D.; Young, A. F.; Yankowitz, M.; Leroy, B. J.; Watanabe, K.; Taniguchi, T.; Moon, P.; Koshino, M.; Jarillo-Herrero, P.; Ashoori, R. C. Science 2013, 340, 1427-1431.

(17) Wallbank, J. R.; Mucha-Kruczyński, M.; Chen, X.; Fal'ko, V. I. Ann. Phys. 2015, 527, 359-376.

(18) Wallbank, J. R.; Patel, A. A.; Mucha-Kruczyński, M.; Geim, A. K.; Fal'Ko, V. I. Phys. Rev. B: Condens. Matter Mater. Phys. 2013, 87, 245408.

(19) Cho, Y.; Summerfield, A.; Davies, A.; Cheng, T. S.; Smith, E. F.; Mellor, C. J.; Khlobystov, A. N.; Foxon, C. T.; Eaves, L.; Beton, P. H.; Novikov, S. V. Sci. Rep. 2016, 6, 34474.

(20) Vuong, P.; Cassabois, G.; Valvin, P.; Rousseau, E.; Summerfield, A.; Mellor, C.; Cho, Y.; Cheng, T.; Albar, J. D.; Eaves, L.; Foxon, C.; Beton, P.; Novikov, S.; GIL, B. 2D Mater. 2017, 4, 021023 .

(21) Cheng, T. S.; Summerfield, A.; Mellor, C. J.; Davies, A.; Khlobystov, A. N.; Eaves, L.; Foxon, C. T.; Beton, P. H.; Novikov, S. V. J. Vac. Sci. Technol., B: Nanotechnol. Microelectron.: Mater., Process., Meas., Phenom. 2018, 36, $02 \mathrm{D} 103$.
(22) Britnell, L.; Gorbachev, R. V.; Geim, A. K.; Ponomarenko, L. A.; Mishchenko, A.; Greenaway, M. T.; Fromhold, T. M.; Novoselov, K. S.; Eaves, L. Nat. Commun. 2013, 4, 1794-1795.

(23) Amet, F.; Williams, J. R.; Garcia, A. G. F.; Yankowitz, M.; Watanabe, K.; Taniguchi, T.; Goldhaber-Gordon, D. Phys. Rev. B: Condens. Matter Mater. Phys. 2012, 85, 73405.

(24) Britnell, L.; Gorbachev, R. V.; Jalil, R.; Belle, B. D.; Schedin, F.; Katsnelson, M. I.; Eaves, L.; Morozov, S. V.; Mayorov, A. S.; Peres, N. M. R.; Castro Neto, A. H.; Leist, J.; Geim, A. K.; Ponomarenko, L. A.; Novoselov, K. S. Nano Lett. 2012, 12, 1707-1710.

(25) Vdovin, E. E.; Mishchenko, A.; Greenaway, M. T.; Zhu, M. J.; Ghazaryan, D.; Misra, A.; Cao, Y.; Morozov, S. V.; Makarovsky, O.; Fromhold, T. M.; Patanè, A.; Slotman, G. J.; Katsnelson, M. I.; Geim, A. K.; Novoselov, K. S.; Eaves, L. Phys. Rev. Lett. 2016, 116, 186603.

(26) Mishchenko, A.; Tu, J. S.; Cao, Y.; Gorbachev, R. V.; Wallbank, J. R.; Greenaway, M. T.; Morozov, V. E.; Morozov, S. V.; Zhu, M. J.; Wong, S. L.; Withers, F.; Woods, C. R.; Kim, Y.-J.; Watanabe, K.; Taniguchi, T.; Vdovin, E. E.; Makarovsky, O.; Fromhold, T. M.; Fal'ko, V. I.; Geim, A. K.; Eaves, L.; Novoselov, K. S. Nat. Nanotechnol. 2014, 9, 808-813.

(27) Greenaway, M. T.; Vdovin, E. E.; Mishchenko, A.; Makarovsky, O.; Patane, A.; Wallbank, J. R.; Cao, Y.; Kretinin, A. V.; Zhu, M. J.; Morozov, S. V.; Fal'ko, V. I.; Novoselov, K. S.; Geim, A. K.; Fromhold, T. M.; Eaves, L. Nat. Phys. 2015, 11, 1057-1062.

(28) Gaskell, J.; Eaves, L.; Novoselov, K. S.; Mishchenko, A.; Geim, A. K.; Fromhold, T. M.; Greenaway, M. T. Appl. Phys. Lett. 2015, 107, 103105.

(29) Feenstra, R. M.; Jena, D.; Gu, G. J. Appl. Phys. 2012, 111, 043711.

(30) Pizzocchero, F.; Gammelgaard, L.; Jessen, B. S.; Caridad, J. M.; Wang, L.; Hone, J.; Bøggild, P.; Booth, T. J. Nat. Commun. 2016, 7, 11894.

(31) Kim, K.; Yankowitz, M.; Fallahazad, B.; Kang, S.; Movva, H. C. P.; Huang, S.; Larentis, S.; Corbet, C. M.; Taniguchi, T.; Watanabe, K.; Banerjee, S. K.; Leroy, B. J.; Tutuc, E. Nano Lett. 2016, 16, 19891995.

(32) Castellanos-Gomez, A.; Buscema, M.; Molenaar, R.; Singh, V.; Janssen, L.; van der Zant, H. S. J.; Steele, G. A. 2D Mater. 2014, 1, 011002 .

(33) Zomer, P. J.; Guimaraes, M. H. D.; Brant, J. C.; Tombros, N.; Van Wees, B. J. Appl. Phys. Lett. 2014, 105, 013101.

(34) Caldwell, J. D.; Anderson, T. J.; Culbertson, J. C.; Jernigan, G. G.; Hobart, K. D.; Kub, F. J.; Tadjer, M. J.; Tedesco, J. L.; Hite, J. K.; Mastro, M. A.; Myers-Ward, R. L.; Eddy, C. R.; Campbell, P. M.; Gaskill, D. K. ACS Nano 2010, 4, 1108-1114.

(35) Lynch, R. W.; Drickamer, H. G. J. Chem. Phys. 1966, 44, 181184.

(36) Heilmann, M.; Bashouti, M.; Riechert, H.; Lopes, J. M. J. 2D Mater. 2018, 5, 025004.

(37) Tang, S.; Wang, H.; Wang, H. S.; Sun, Q.; Zhang, X.; Cong, C.; Xie, H.; Liu, X.; Zhou, X.; Huang, F.; Chen, X.; Yu, T.; Ding, F.; Xie, X.; Jiang, M. Nat. Commun. 2015, 6, 6499.

(38) Yang, W.; Chen, G.; Shi, Z.; Liu, C.-C.; Zhang, L.; Xie, G.; Cheng, M.; Wang, D.; Yang, R.; Shi, D.; Watanabe, K.; Taniguchi, T.; Yao, Y.; Zhang, Y.; Zhang, G. Nat. Mater. 2013, 12, 792-797.

(39) Davies, A.; Albar, J. D.; Summerfield, A.; Thomas, J. C.; Cheng, T. S.; Korolkov, V. V.; Stapleton, E.; Wrigley, J.; Goodey, N. L.; Mellor, C. J.; Khlobystov, A. N.; Watanabe, K.; Taniguchi, T.; Foxon, C. T.; Eaves, L.; Novikov, S. V.; Beton, P. H. Nano Lett. 2018, 18, 498-504.

(40) Amorim, B.; Ribeiro, R. M.; Peres, N. M. R. Phys. Rev. B: Condens. Matter Mater. Phys. 2016, 93, 235403. 